\title{
La prévision des précipitations : progrès depuis dix ans, perspectives à dix ans
}

\author{
Precipitation forecasting : \\ 10 years of progress, perspectives for the 10 next years
}

par Pierre Bénard

Météo-France - CNRM/GMAP/MOD

Meteo-France has two main tools for forecasting precipitations : 2PIR, as a nowcasting tool, and a panoply of numerical models (IFS, ARPEGE, Aladin) for short to medium range forecast.

As in every National Meteorological Services the quality of the numerically forecasted precipitations is routinely evaluated at Meteo-France. The particular variability of this field imposes to evaluate the accumulated precipitations against observed values themselves.

The quality of the forecasted precipitations has been improved significantly since ten years, and is comparable roughly with the quality obtained at ECMWF (Reading, UK), one of the leading Centres in the world. These improvements can mainly be explained by the refinement of the horizontal resolution allowed by the available computers capacity, and in a lesser extend by progresses in the forecast technique itself (dynamical and physical representation, data assimilation).

The perspectives to ten years are far from being vanishing. The future better results should come from progresses in the same three main ingredients (further realism in the physical description, further refinement of the horinzontal resolution, and above all, implementation of $4 D$-Var data assimilation with a possibility of real-time modification of the initial state).

\section{INTRODUCTION}

Actuellement la prévision des précipitations à Météo-France et dans les autres Services Météorologiques opérationnels à travers le monde repose essentiellement sur des méthodes de prévision immédiate pour les échéances les plus courtes (jusqu'à moins de six heures) et sur des méthodes de prévision numérique déterministe pour les échéances plus longues (jusqu'à cinq jours en France avec le modèle ARPEGE, dix jours au Centre Européen de Prévisions Météorologiques à Moyen Terme avec le modèle IFS). Pour ces deux types d'échéances, le processus d'obtention de prévisions finalisées est similaire : un outil automatique fournit d'abord une prévision quantitative dans l'espace et dans le temps, et ensuite, le prévisionniste adapte cette prévision en fonction d'autres éléments d'information, comme sa propre connaissance des caractéristiques régionales, ou des informations en provenance d'autres outils ou données observées...
L'estimation de la qualité des prévisions est une tâche vitale pour les Services Météorologiques, et elle est donc généralement effectuée en routine sous le nom de "vérification " ou de "contrôle ». Toutefois, il faut bien garder à l'esprit que cette vérification porte dans l'immense majorité des cas sur la première étape, purement automatique, du processus évoqué ci-dessus, restriction peu handicapante pour notre propos, puisque les applications hydrologiques sont généralement insérées en amont de l'expertise du prévisionniste.

D'une manière similaire, la " vérité " utilisée pour estimer la qualité des prévisions est choisie la moins arbitraire possible : on utilise donc les données observées et les états équilibrés de l'atmosphère à l'échelle de la maille du modèle, obtenus numériquement à partir de ces observations et appelés « états analysés ». On parle donc de " contrôle objectif ", le travail du prévisionniste dans son ensemble étant considéré comme « subjectif ».

Enfin, il faut souligner que excepté quelques études spécifiques, le contrôle objectif des précipitations prévues ne fait en général pas de distinction entre les phénomènes 
" hydrologiquement dangereux " et les autres. Les chiffres donnés ici concernent donc sauf exception la qualité de prévision des précipitations en général et non pas la qualité de prévision des fortes précipitations.

\section{LES OUTILS DISPONIBLES}

Les outils disponibles actuellement à Météo-France pour la prévision des précipitations sont: le système 2PIR de prévision immédiate entre 0 et quelques heures d'échéance, et le système de prévision numérique bâti autour des modèles ARPEGE, IFS et ALADIN, entre 12 et 96 heures d'échéance.

Le système 2PIR est basé sur l'extrapolation automatique du déplacement des échos RADAR, une méthode qui, aux échelles spatiales visées, est applicable pour des échéances temporelles inférieures à quelques heures. Dans la version actuellement disponible, le calcul et la visualisation se font pour un site RADAR unique. C'est donc principalement la limitation de la zone de détection qui impose l'échéance exploitable, ceci à environ une heure et demie. En tant qu'outil de prévision immédiate, ce système fonctionne de manière autonome sur le site du client. Les précipitations sont converties en taux de précipitation par une loi fixe. Les précipitations ainsi prévues peuvent alors être spatialement intégrées sur des bassins pré-déterminés. Les applications installées auprès des SAC utilisent un outil plus précis pour calculer les lames d'eau à partir des réflectivités RADAR, et baptisé HYDRAM. Toutefois, il faut bien garder à l'esprit que la méthode 2PIR présente des limitations qui restreignent son application : dans les régions au relief accidenté, la détection des échos précipitants est beaucoup moins aisée, et l'extrapolation de la trajectoire des cellules devient sujette à caution, du fait de la canalisation partielle du flux par les reliefs. Le système 2PIR rend donc surtout les plus grands services dans les zones urbaines non-montagneuses où c'est la nature du sol qui entraîne les fortes vitesses d'écoulement.

Le système 2PIR est donc un système de prévision immédiate à échelle fine, surtout susceptible de fournir une aide pour la prévision des crues rapides urbaines.

La méthode de prévision numérique est basée sur l'intégration numérique temporelle des équations discrétisées de la physico-dynamique atmosphérique, à partir d'un état initial optimal du point de vue de l'adéquation aux observations, aux prévisions précédemment obtenues et aux lois qui régissent l'équilibre de l'atmosphère, état lui aussi obtenu numériquement. Les trois systèmes utilisés à Météo-France dans cette optique (IFS, ARPEGE et ALADIN) ont des domaines et/ou des mailles différentes, et sont utilisés à des échéances différentes en accord avec ces dernières. Cette méthode, qui ne permet pas des résolutions extrêmement fines du fait de l'énorme masse de calcul à effectuer, est surtout compétitive aux échéances non-immédiates, avec une limite actuelle vers 96-120 heures. Les produits disponibles pertinents ici sont principalement les cumuls de précipitations sur 6 heures. Cet outil est donc plus particulièrement adapté à la prévision des crues lentes.

Un facteur essentiel pour la prévision des précipitations est la résolution horizontale : l'augmentation de la résolution horizontale permet une meilleure description des phénomènes atmosphériques, mais aussi une meilleure représentation du relief. Le modèle IFS exploité au Centre Européen de Prévisions Météorologiques à Moyen Terme (CEPMMT) est un modèle global qui possède sur la France une résolution de $40 \mathrm{~km}$; le modèle ARPEGE lui aussi global mais à résolution variable atteint une résolution de $20 \mathrm{~km}$ sur la France, tandis que le modèle en aire limitée ALADIN qui lui est couplé atteint $10 \mathrm{~km}$.

\section{III — LES PERFORMANCES ET LEUR ÉVOLUTION DEPUIS 10 ANS}

En ce qui concerne la prévision immédiate par 2PIR, il n'y a pas véritablement d'estimation en routine de la qualité, et parmi les tests de vérification effectués, il existe peu de vérification aux observations, principalement à cause de l'échelle très fine des phénomènes prévus. Le fait que ce produit "échappe " aux contrôles de qualité opérationnels provient probablement du fait qu'aux très courtes échéances, auxquelles il est le plus utilisé, la qualité est suffisamment bonne pour faire l'unanimité. De plus, l'essence même de la méthode utilisée n'offre pas de grande évolution possible, et les performances sont grosso-modo constantes à travers les années. Les graphes de corrélation (réflectivité prévue/observée) montrent systématiquement une bonne corrélation entre prévision et observation pour des échéances inférieures à une heure. La faible dispersion autour de l'axe médian pour les grandes valeurs de réflectivité est de plus une caractéristique très intéressante pour les hydrologues. Toutefois, comme mentionné ci-dessus, les performances se dégradent rapidement en présence de relief, et le système est peu utilisé pour les crues non-urbaines.

En ce qui concerne la prévision à plus longue échéance, de nombreuses validations sont effectuées en routine, et nous n'en aborderons que les points essentiels, en les illustrant par des exemples représentatifs.

Tout d'abord, la répartition spatiale des précipitations prévues par les modèles est généralement à peu près satisfaisante, à l'échelle du trimestre et même du mois, et le total global des précipitations est réaliste. En revanche, on note souvent une erreur locale sur la valeur des cumuls, notamment sur les reliefs (cf. fig. 1), dans le sens d'une surestimation des précipitations. Les tables de contingences par classes de phénomènes (cf. tableaux 1 et 2) montrent aussi en général une surestimation des cas de pluies faibles lorsque les pluies observées sont nulles. Ces défauts sont communs, à des degrés divers, à tous les modèles utilisés à Météo-France.

L'évolution des scores de prévision pour les précipitations peut être résumé ainsi : la qualité des prévisions de précipitation sur la France a été améliorée d'un facteur 2 depuis 10 ans (cf. fig. 2), les écarts-types d'erreur aux observations du cumul en 6 heures passant de $3 \mathrm{~mm}$ à $1,5 \mathrm{~mm}$ pour les prévisions à 24 heures. La surveillance systématique des performances a aussi permis de se donner les moyens de diminuer considérablement les biais sur le territoire français.

Les performances actuelles des modèles français par rapport à leurs homologues d'autres pays peuvent être illustrées sur les comparaisons avec les performances du modèle du CEPMMT, considéré comme l'un des meilleurs modèles mondiaux de prévision (cf. fig. 3). Les écarts quadratiques moyens (EQM) aux observations pour les cumuls en 6 heures sont tout à fait comparables pour les deux modèles, ainsi que l'ordre de grandeur des biais, ce qui montre que les deux modèles possèdent un niveau comparable de performance. D'une manière plus générale, on peut dire que les modèles de Météo-France se placent dans le peloton (assez groupé) de l'ensemble des grands modèles européens et mondiaux, pour les scores de prévisions de précipitations.

Toutefois, il faut souligner que le champ de précipitations est l'un des plus difficiles à prévoir pour un modèle météorologique, du fait de sa très grande variabilité spatiale et temporelle. Même si des progrès importants ont été réalisés depuis ces dernières années, ce champ reste tout de même, pour l'ensemble des modèles mondiaux, l'un des plus mal prévus, en comparaison de champs plus « lisses " comme le vent, la température, etc.

En résumé, pour les modèles numériques, il y a donc une amélioration générale dans les prévisions de précipitations, et Météo-France se situe au bon niveau dans la compétition mondiale ou européenne. 


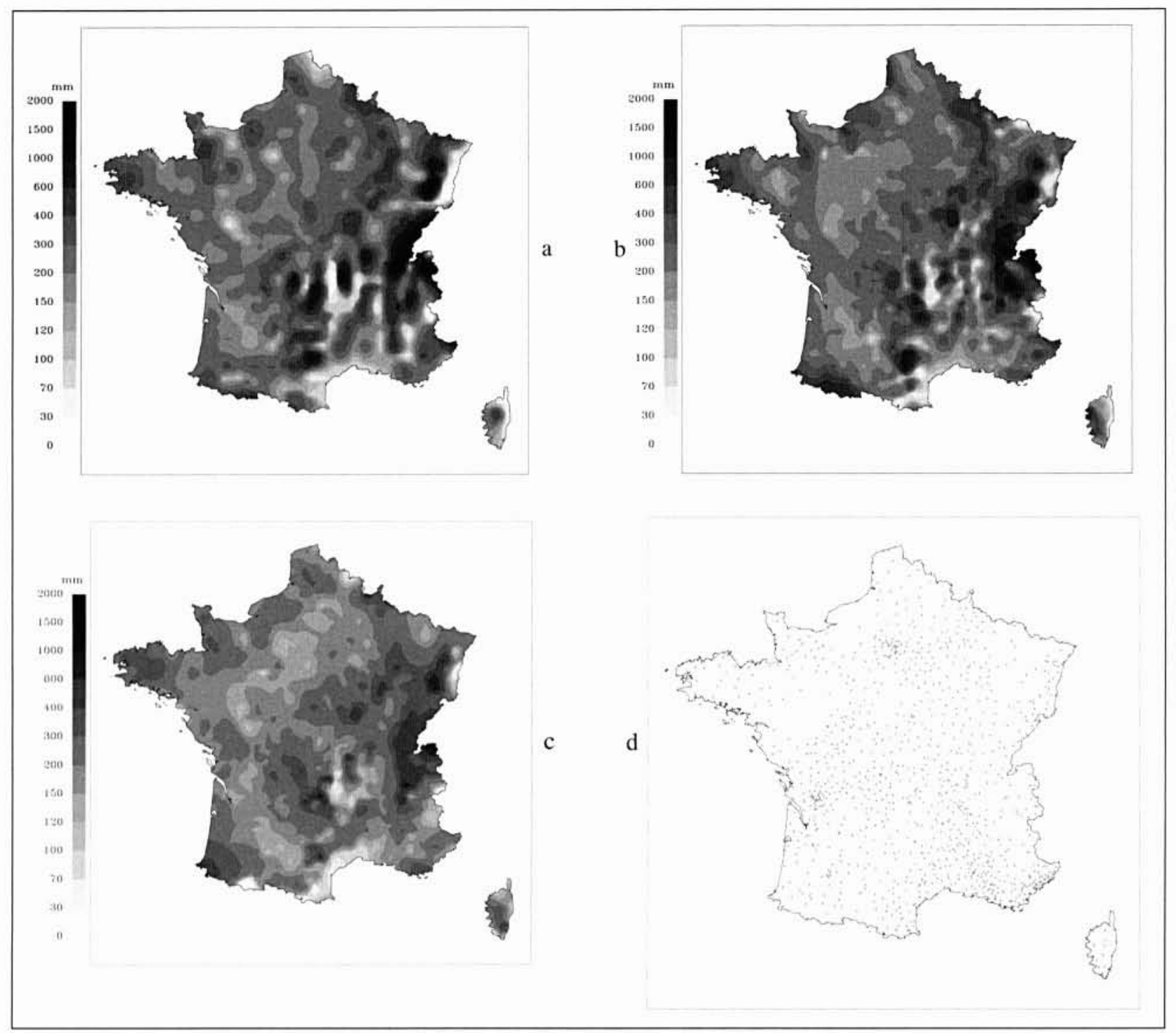

1. Cumuls de précipitations prévues (entre 06 et 30 heures d'échéance) sur la France (à partir de $00 \mathrm{TU}$ ) pour le $1 \mathrm{er}$ trimestre 1999 ramenés aux stations d'observations principales (a) ARPEGE, (b) Aladin, (c) Observations, (d) répartition des postes d'observation.

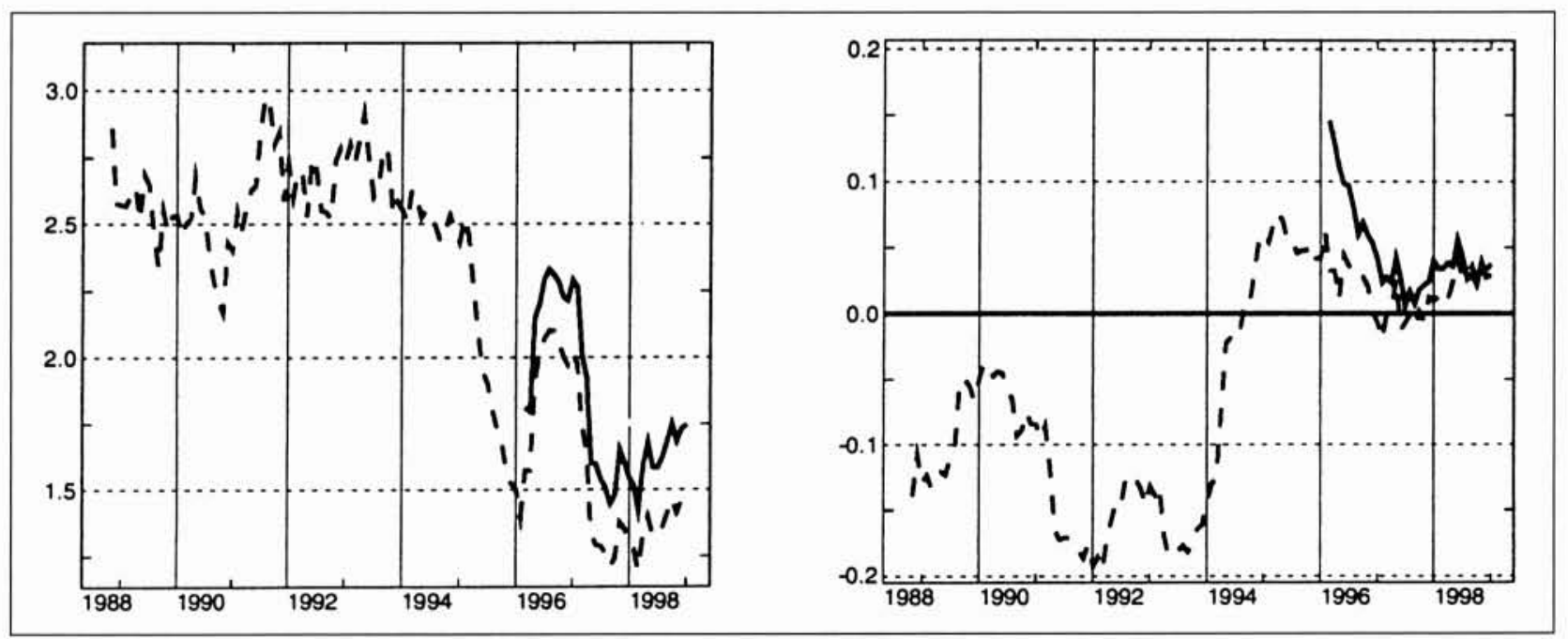

2. Evolution (en $\mathrm{mm}$ ) de l'écart-type (gauche) et du biais (droite) des erreurs de prévision du cumul de précipitations en 6 heures comparés aux observations, pour le modèle ARPEGE à 24 heures (trait tireté) et 72 heures (trait plein), en moyenne glissante sur un an de 1988 à 1999. 
Tableau 1. - Table de contingence (en \%) des précipitations ARPEGE (réseau 00 UTC) à échéance $18 \mathrm{~h}$ sur la France, pour les mois d'octobre 1997 à mars 1998. Interpolation des précipitations observées au point de grille terrestre le plus proche (maille 0,25 degrés). Total 28407 observations.

\begin{tabular}{|c|c|c|c|c|c|c|}
\hline & \multicolumn{7}{|c|}{ Précipitations prévues } \\
\hline \multirow{4}{*}{$\begin{array}{c}\text { Précipitations } \\
\text { observées }\end{array}$} & Nulles & 64.3 & 13.4 & 1.6 & 0.1 & 79.4 \\
\cline { 2 - 8 } & Faibles & 2.6 & 6.2 & 2.2 & 0.1 & 11.1 \\
\cline { 2 - 8 } & Modérées & 0.7 & 3.6 & 3.8 & 0.5 & 8.5 \\
\cline { 2 - 7 } & Fortes & 0.1 & 0.2 & 0.4 & 0.3 & 0.9 \\
\cline { 2 - 8 } & Total & 67.7 & 23.4 & 8.0 & 0.9 & \\
\hline
\end{tabular}

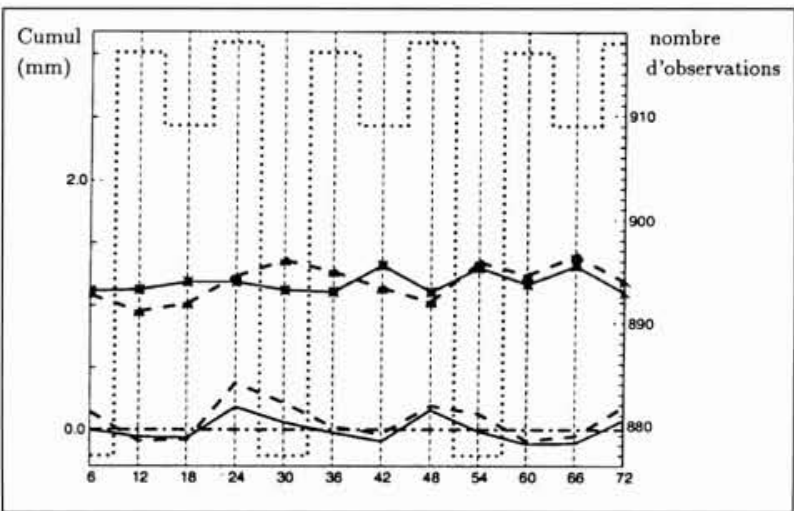

3. Comparaison des scores aux observations en écart-type et biais du modèle ARPEGE (Météo-France) et du modèle IFS (CEPMMT) pour les cumuls en 6 heures sur le domaine français. Abcisse : Echéance de prévision. Ordonnée: Cumul en 6 heures (gauche), nombre d'observations (droite). Trait plein : ARPEGE ; trait tireté : CEPMMT ; trait pointillé : nombre d'observations. Ecarttype : courbes du haut ; biais : courbes du bas.3

\section{FACTEURS DES PROGRÈS RÉA- LISÉS}

Seule la méthode de prévision numérique est abordée ici. Les progrès réalisés depuis dix ans peuvent s'analyser de manière assez simple par deux principaux facteurs : l'augmentation de la résolution des modèles d'une part, et l'utilisation de schémas de paramétrisations plus sophistiqués pour décrire les différents processus physiques à l'œuvre dans l'atmosphère d'autre part.

La résolution la plus fine utilisée à Météo-France est passée de $35 \mathrm{~km}$ à $10 \mathrm{~km}$ dans l'intervalle. A cette échelle, l'organisation interne de certains systèmes structurés commence à être décrite, bien que les échelles les plus communes de la convection (de l'ordre de quelques $\mathrm{km}$ ) ne le soient pas encore. Ces systèmes structurés étant évidemment plus violents que des systèmes isolés de moindre amplitude, l'augmentation de résolution permet surtout un gain dans ces gammes de phénomènes. Cela est renforcé par le fait que l'orographie est mieux décrite à échelle fine, ainsi donc que ses interactions avec l'atmosphère. Des phénomènes comme les crues de Vaison-la-Romaine en 1992 n'auraient pas pu être prévus correctement avec les résolutions d'il y a dix ans alors que cela est possible avec les résolutions actuelles.

L'évolution des modèles numériques va sans cesse dans le sens d'une plus grande complexité dans la description des
Tableau 2. - Table de contingence (en $\%$ ) des précipitations Aladin (réseau 00 UTC) à échéance $36 \mathrm{~h}$ sur la France, pour les mois d'octobre 1997 à mars 1998. Interpolation des précipitations observées au point de grille terrestre le plus proche (maille 0,1 degrés). Total 27633 observations.

\begin{tabular}{|c|c|c|c|c|c|c|}
\hline & \multicolumn{7}{|c|}{ Précipitations prévues } \\
\hline \multirow{4}{*}{$\begin{array}{c}\text { Précipitations } \\
\text { observées }\end{array}$} & Nulles & 64.4 & 9.3 & 1.8 & 0.2 & 75.5 \\
\cline { 2 - 7 } & Faibles & 6.8 & 5.4 & 2.7 & 0.2 & 15.0 \\
\cline { 2 - 7 } & Modérées & 1.3 & 3.2 & 3.5 & 0.5 & 8.5 \\
\cline { 2 - 7 } & Fortes & 0.1 & 0.3 & 0.4 & 0.2 & 0.9 \\
\cline { 2 - 7 } & Total & 72.5 & 18.1 & 8.3 & 1.1 & \\
\hline
\end{tabular}

phénomènes physiques. Depuis une dizaine d'années les avancées majeures ont surtout concerné la description des échanges de chaleur et de vapeur d'eau au sol, des transferts dynamiques verticaux non-résolus au dessus des reliefs et des schémas de microphysique et de convection. Pour le premier point, on est passé en dix ans d'une physique très simplifiée de l'interface sol-biosphère-atmosphère à des schémas prenant en compte de manière complexe les phénomènes d'interception, d'évapo-transpiration, de drainage, d'écoulement, etc. tenant compte à la fois de la nature des sols et de leur réservoirs, mais aussi de celle de la végétation. La mise en place de ces schémas s'est naturellement accompagnée d'une analyse plus réaliste des variables météorologiques au sol, domaine qui est en pleine phase ascendante actuellement et dont l'impact sur la qualité des précipitations, bien qu'indirect, est probablement non négligeable.

Enfin, la qualité des prévisions de précipitations a aussi bénéficié, dans une moindre mesure, des progrès concernant les techniques d'assimilation de données, et les données elles-mêmes. Les observations satellitaires et celles des réseaux automatiques au sol sont mieux intégrées au calcul de l'état initial de la prévision, bien qu'il soit difficile de chiffrer l'impact final de ces avancées pour les précipitations elles-mêmes.

\section{E ÉVOLUTIONS PLANIFIÉES COURT ET MOYEN TERMES}

Pour la prévision aux courtes échéances, l'amélioration du produit 2PIR est la principale évolution en vue : l'extension du produit non plus à un site RADAR unique, mais à l'ensemble de la mosaïque permettra de reculer l'échéance exploitable de 90 minutes à quelques heures (en gardant à l'esprit que la méthode possède une limitation intrinsèque vers 6 heures, au-delà de laquelle son apport bénéfique disparaît). Par contre les tentatives pour ajouter une extrapolation de l'intensité à celle de la trajectoire ont été infructueuses : l'évolution en intensité des systèmes convectifs, gouvernée par leur physique interne, est plus chaotique que leur trajectoire, gouvernée principalement par le flux environnemental, assez régulier.

On envisage également d'adopter une méthode similaire à celle utilisée au Service Météorologique de Grande-Bretagne (UKMO) pour combler le "trou de prévision " actuel entre environ 1 heure 30 et 12 heures d'échéance. Leur système, baptisé NIMROD, établit des champs de précipitations prévus grâce à des algorithmes d'interpolations entre champs issus de la prévision immédiate et de la prévision numérique.

D'autres méthodes prédictives pour les précipitations peuvent être employées pour les courtes échéances, et sont envi- 
sagées dans le cadre de la production vers le grand public, mais leur utilisation à des fins hydrologiques semble plus problématique. Ces méthodes recouvrent d'une part l'estimation d'un indice du risque de convection sévère en fonction des observations au sol et des profils issus des modèles numériques, mais ceci ne permet pas de déboucher sur une prédiction quantitative des flux de précipitation; d'autre part l'adaptation statistique des précipitations prévues grâce à un apprentissage statistique sur des échantillons historiques, mais ici le raffinement des prévisions concerne uniquement les stations de mesure, pour lesquelles on dispose de séries temporelles d'observations, et ne peut donc être spatialisé.

Pour les modèles numériques, les avancées planifiées à assez court terme et susceptibles d'améliorer les prévisions de précipitations sont multiples :

a) Poursuite des efforts en résolution, grâce notamment à l'installation d'une machine de calcul plus performante au début de l'année prochaine.

b) Poursuite des efforts en finesse de description de l'interface sol-biosphère-atmosphère, avec l'incorporation de données fines concernant les sols et la végétation, sur l'ensemble du globe. L'ajout de couches supplémentaires de réservoirs dans le sol, notamment pour mieux décrire les phases de gel/dégel permettra un meilleur réalisme pour les champs aux bas niveaux, et donc également pour les flux au sol. Les efforts seront naturellement poursuivis pour améliorer l'analyse des champs au sol.

c) Saut qualitatif dans la sophistication du schéma microphysique utilisé, avec le recours à des variables pronostiques pour les phases condensées de l'eau atmosphérique, et une refonte en profondeur des différents schémas d'interaction entre phases.

d) Poursuite des efforts dans l'utilisation des données satellitaires, afin de mieux initialiser la structure verticale de l'atmosphère notamment sur les océans.

e) Test d'une modification de l'analyse de la température de surface de la mer (SST en anglais) en incluant un terme de rappel vers l'analyse en temps réel effectué par le service américain NESDIS au lieu du rappel actuel vers la SST climatologique.

Mentionnons de plus que le choix stratégique (et crucial) de représenter finement la physique de l'interface sol-biosphère-atmosphère dans les modèles de prévision météorologiques impose presque automatiquement pour nous la nécessité de développer l'élaboration de produits hydrologiques au travers du couplage avec des modèles hydrologiques. Cette nécessité vient du fait que les flux hydrologiques sont des informations de première importance pour la validation in fine de ces schémas physiques, et leur suivi opérationnel. Les débits aux exutoires des bassins versants deviennent alors pour nous des variables de contrôle permettant de monitorer et valider la pertinence nos schémas de sol (qui rappelons-le fournissent notamment des flux de ruissellement) par comparaison aux débits observés.

\section{VI $\square$ PERSPECTIVES À LONG TERME}

Pour le plus long terme, les perspectives peuvent s'interpréter à la lumière des objectifs scientifiques de Météo-France en matière de Prévision Numérique à l'échéance de dix ans : la disposition d'un système de prévision opérationnel à échelle fine (typiquement 2 kilomètres) avec dynamique non-hydrostatique (donc avec convection résolue explicitement), physique sèche et micro-physique détaillées, assimilation variationnelle continue des observations (méthode dite 4D-VAR) incluant non seulement l'ensemble des données télédétectées disponibles, mais aussi permettant l'introduction de données d'observation directes en temps réel afin de modifier les prévisions à moyenne échelle.
L'utilisation de modèles non-hydrostatiques à résolution fine est reconnue depuis longtemps comme permettant un saut qualitatif important pour la prévision des phénomènes liés aux précipitations non stratiformes. Le fait que la convection devienne résolue explicitement (au moins en grande partie) permet de s'affranchir des schémas de paramétrisation de la convection, simplificateurs par essence.

De plus, à de telles résolutions, l'organisation interne de la plupart des systèmes convectifs organisés (donc violents) est également décrite, ce qui est un atout décisif pour espérer prévoir leur intensité et leur évolution (ces systèmes ont souvent tendance à s'alimenter eux-mêmes en interagissant avec le flux).

Les schémas de paramétrisation des flux turbulents dans l'atmosphère libre et au voisinage du sol sont également appelés à d'importants progrès. Ceux-ci nécessiteront la mise en œuvre de schémas de paramétrisation de la turbulence utilisant une (ou deux) variables pronostiques supplémentaire(s) pour représenter l'énergie cinétique turbulente (et la dissipation). Ces schémas d'un ordre de précision plus élevé que ceux utilisés actuellement permettront une meilleure description des échanges au sol et dans la couche limite de l'atmosphère, fondamentaux pour la prévision des précipitations même si c'est de manière indirecte. Les phénomènes de convection peu profonde seront probablement résolus explicitement eux aussi, au moins en partie.

Les progrès dans l'acquisition de ces données observationnelles et dans les méthodes d'assimilation de ces données profiteront aussi évidemment aux prévisions de précipitation. Les données satellitaires de profils verticaux seront exploitées en routine sur tout le globe et pourront être assimilées même si elles ne sont reliées qu'indirectement aux variables du modèle, du fait de l'utilisation de méthodes variationnelles, et elles le seront de manière continue dans le temps du fait de la technique d'assimilation 4D-VAR qui fournit un état initial en optimisant continûment la trajectoire du modèle par rapport à l'ensemble des observations passées. On peut également penser que les données RADAR seront au moins partiellement utilisées en assimilation dans les modèles d'échelle fine.

Enfin, pour les modèles d'échelle fine, une possibilité de modifier en temps réel l'état initial par des observations jugées pertinentes et de lancer immédiatement une nouvelle prévision est actuellement à l'étude. Cette méthode dite "bogussing " consiste donc en fait à effectuer une assimilation simplifiée suivie immédiatement d'une prévision.

On devrait enfin également assister à un développement des méthodes de prévision non déterministes comme la Prévision d'Ensemble. Ces méthodes, qui consistent à effectuer un échantillon de plusieurs prévisions avec des états initiaux légèrement différents, restent quantitatives comme les prévisions déterministes actuelles, mais fournissent des probabilités pour chaque classe de valeurs pour les paramètres prévus.

Il est bien difficile d'évaluer quel sera l'impact de l'ensemble de ces avancées en termes de perspectives pour les prévisions de précipitations elles-mêmes. Toutefois, il est certain que ce tableau de perspectives se donne clairement pour ambition de prévoir les épisode paroxystiques qui intéressent les hydrologues. Dire que les épisodes comme Vaison-la-Romaine seront quantitativement prévus à coup sûr relèverait d'un optimisme excessif, mais les ingrédients fondamentaux pour le succès de ce type de prévisions seront là. Pour Vaison, par exemple, nous savons que le phénomène peut être correctement prévu, même avec les versions actuelles des modèles, pourvu que le champ d'humidité sur la mer Méditerranée au voisinage des îles Baléares soit correctement initialisé (ce qui n'avait pas été le cas à l'époque, faute de données). Ce qui fait le plus défaut dans ce type de cas c'est donc souvent une couverture généralisée de données fiables sur les mers et océans. Le tableau des objectifs scientifiques ci-dessus semble donc suffisant pour répondre à la 
question dans ce cas précis, mais il restera toujours d'autres cas où des causes très ténues conduiront à des prévisions erronées. Pour ne citer qu'un exemple, le cas des situations orageuses où les cellules n'ont pas d'organisation particulière restera extrêmement difficile à prévoir correctement autrement qu'en termes probabilistes. Il est pratiquement certain que la prévision numérique dans dix ans ne sera pas un outil tellement meilleur qu'aujourd'hui pour la prévision d'une crue rapide particulière issue d'un système non organisé.

En ce qui concerne les méthodes de prévision immédiate telles qu'implantées actuellement, on peut dire qu'elles sont pratiquement condamnées à long terme, ceci pour deux raisons principales: d'une part la base méthodologique sur laquelle elles reposent (extrapolation pure et simple des champs dans l'ignorance totale de la physique des phénomènes) n'est pas évolutive et donc les résultats ne sont pas susceptibles d'amélioration significative dans le temps. D'autre part, le «trou » de prévision pour les échéances en deçà de 12 heures mentionné ci-dessus disparaîtra lorsque la technique d'assimilation en continu 4D-VAR sera appliquée, et les très courtes échéances seront donc accessibles à la prévision numérique elle-même. En effet, le trou en question est dû au fait qu'avec les techniques d'assimilations intermittentes actuelles (toutes le 6 heures en pratique), l'introduction des données crée un choc dans les conditions initiales, qui nécessite environ 6 à 12 heures pour être complètement résorbé par le modèle. Durant cet intervalle, les prévisions sont sujettes à caution et ne sont pas utilisées. Avec les techniques d'assimilation continue, on pourra disposer à chaque instant d'un état du modèle qui soit en accord avec toutes les observations passées et équilibré au sens des équations dynamiques, donc prêt à démarrer sans aucun choc. Dans ces conditions, il est possible de lancer des prévisions à courte échéance à tout moment. Le seul obstacle devient alors celui du coût de l'opération, qui impose de définir une politique claire à ce sujet. Pour la prévision à courte échéance, c'est donc la prévision numérique qui prendra logiquement le relais des méthodes de prévision immédiate actuelles.

\section{VII $\square$ CONCLUSIONS}

La prévision des précipitations a significativement progressé ces dix dernières années, avec l'avènement de la prévision immédiate pour les très courtes échéances, et une amélioration d'un facteur deux en moyenne pour la prévision aux échéances intermédiaires de moins de 5 jours (constat positif à tempérer probablement car il s'agit de scores sur tous les événements pluvieux, et non pas spécifiquement sur les événements donnant lieu à des crues). De plus, la France se situe à une place honorable internationalement pour ce qui est de la qualité des prévisions de précipitations.

Les facteurs de cette amélioration sont assez bien cernés et concernent surtout la résolution, le réalisme des paramétrisations, la couverture des données et l'assimilation de ces données.

Les perspectives à court terme et à plus long terme reprennent ces problématiques et tentent de dégager les axes où les progrès les plus significatifs sont susceptibles d'être atteints. Les étapes décisive viendront probablement du passage à des résolutions beaucoup plus élevées, avec pour corollaire la relaxation de l'hypothèse hydrostatique, et la modification des paramétrisations physiques pertinentes pour ces échelles, de la généralisation de l'utilisation de données télédétectées sur les zones pauvres en observations conventionnelles, et du passage à l'assimilation $4 \mathrm{D}-\mathrm{VAR}$, qui permettra en plus de l'amélioration des prévisions, d'aborder la prévision à courte échéance sur des bases scientifiques plus solides qu'actuellement.

Dans dix ans, on peut espérer que plus aucun service opérationnel en Europe ne se fera surprendre par une crue lente non prévue; en revanche, même si des progrès importants sont réalisés, la prévision des crues rapides comprendra toujours une part de hasard non maitrisable à cette échéance. 